\title{
An Analytical Expression for Drain Saturation Voltage of Polycrystalline Silicon Thin-Film Transistors
}

\author{
Han Hao, Mingxiang Wang, Member, IEEE, and Man Wong, Senior Member, IEEE
}

\begin{abstract}
A physical-based analytical expression for the drain saturation voltage $V_{\text {Dsat }}$ of polycrystalline silicon (poly-Si) thinfilm transistors (TFTs) is derived. $V_{\text {Dsat }}$ is found to be dominated by the grain boundary potential barrier modulation effect, which can be readily estimated from the device transfer characteristic. Straightforward prediction of $V_{\text {Dsat }}$ values at arbitrarily given gate voltages based on the proposed formula is demonstrated for both low temperature and high temperature processed poly-Si TFTs in either n- or p-type. The prediction agrees well with experimentally determined $V_{\text {Dsat }}$ value. Derivation of the expression is based on our previously proposed analytical oN-state drain-current model for poly-Si TFTs, with no empirical factors included.
\end{abstract}

Index Terms-Drain saturation voltage, output characteristic, polycrystalline silicon, thin-film transistors (TFTs).

\section{INTRODUCTION}

W ITH THE rapid development of new polycrystalline silicon thin-film transistor (poly-Si TFT) technologies, TFT device modeling [1]-[6] becomes increasingly important to practical applications of poly-Si TFT-based circuits. Drain saturation voltage $V_{\text {Dsat }}$ is one of the key parameters for modeling of device output characteristics. For "ideal" MOSFETs, $V_{\text {Dsat }}=V_{g}-V_{t} \equiv V_{\mathrm{gt}}$, where $V_{t}$ is the gate bias when channel inversion just occurs. By including some first-order effects in MOSFETs, namely carrier velocity saturation and transverse field $\left(E_{\text {eff }}\right)$ dependent mobility degradation effect, analytical formulas are derived for $V_{\text {Dsat }}$ prediction [7]-[9]. These formulas are equivalent and calculated $V_{\text {Dsat }}$ values are close. Sometimes for simplicity, a "saturation factor" $\alpha$ is introduced to define $V_{\text {Dsat }}$ by $V_{\text {Dsat }}=V_{\text {gt }} / \alpha$ [8], [9]. $\alpha$ equals 1 for "ideal" MOSFETs and becomes slightly larger than 1 due to the nonideal first-order effects. Here, the physical basis of the saturation factor is clear.

For poly-Si TFTs, however, there is still no generally accepted analytical formula for $V_{\text {Dsat }}$ prediction. Instead, some previous studies [1], [2] empirically introduced the saturation factor $\alpha$ as a fitting parameter for $V_{\text {Dsat }}$ modeling. Without

Manuscript received November 19, 2007; revised January 23, 2008. This work was supported by the National Natural Science Foundation of China under the Contract 60406001 . The review of this letter was arranged by Editor Y. Taur.

H. Hao and M. Wang are with the Department of Microelectronics, Soochow University, Suzhou 215021, China (e-mail: Mingxiang_wang@suda.edu.cn).

M. Wong is with the Department of Electronic and Computer Engineering, The Hong Kong University of Science and Technology, Kowloon, Hong Kong.

Color versions of one or more of the figures in this letter are available online at http://ieeexplore.ieee.org.

Digital Object Identifier 10.1109/LED.2008.917810 knowing the physical basis of $\alpha$ in TFTs, it can only be obtained by fitting the experimental output curve at some specific $V_{g}$. In practice, $\alpha$ is found to be $V_{g}$ dependent and usually considerably larger than one [2], [6]. Therefore, straightforward prediction of $V_{\text {Dsat }}$ before measuring the corresponding output characteristic is not possible in this approach. In other previous works [3]-[5], without introducing $\alpha, V_{\text {Dsat }}$ was obtained numerically [4], [5] or through an expression very close to that for MOSFETs [3]. With a number of fitting parameters involved there, prediction of $V_{\text {Dsat }}$ was not able to be addressed. Up to now, a physical-based analytical formula enabling the straightforward prediction of $V_{\text {Dsat }}$ of poly-Si TFTs is still absent, and is therefore highly desired.

Recently, we developed a physical-based analytical on current model for poly-Si TFTs [6], where the physical basis of $\alpha$ has been clarified. It is shown that three effects, $V_{g}$ dependent grain boundary (GB) potential barrier modulation, $E_{\text {eff }}$ dependent mobility degradation and carrier velocity saturation contribute to its nonideality. This may provide an approach for straightforward prediction of $V_{\text {Dsat }}$. Unfortunately, the expression for $\alpha$ is a rough estimation that includes four physical parameters. In practice, $\alpha$ still has to be empirically used as a fitting parameter. In this letter, we propose for the first time a physical-based analytical formula enabling the straightforward calculation of poly-Si TFTs' $V_{\text {Dsat }}$ at arbitrarily given $V_{g}$. The given expression contains only two parameters, both of which are physical explicitly defined and can be readily obtained before measuring actual output characteristics. It is derived by further developing our previous model to precisely describe the drain saturation region. Its applicability is demonstrated on different poly-Si TFTs made from various processes.

\section{Drain-Current Model}

At some point $y$ (where the drain voltage drop is $V_{c}$ ) along the inversion channel of poly-Si TFTs, $E_{\text {eff }}$ controlled mobility degradation is modeled as [10]

$$
\mu_{\mathrm{ig}}=\frac{\mu_{0}}{1+\left(E_{\text {eff }} / E_{\text {crit }}\right)^{\nu}}
$$

where $\mu_{0}$ is intra-grain carrier mobility free of surface scattering, and $E_{\text {crit }}$ the critical field for mobility degradation, and $\nu=1$ for holes or 1.6 for electrons [10]. $E_{\text {eff }}$ is controlled by the channel inversion charge sheet density $Q_{\text {inv }}[11]$, [12]

$$
E_{\mathrm{eff}}=\eta Q_{\mathrm{inv}} / \varepsilon_{s}=\eta C_{\mathrm{ox}}\left(V_{\mathrm{gt}}-V_{c}\right) / \varepsilon_{s} .
$$


$\eta$ is $1 / 2$ for electrons and $1 / 3$ for holes [9], [12]. $\varepsilon_{s}$ is the Si permittivity. The intra-grain carrier mobility $\mu_{\text {ig }}$ should be incorporated into a complete effective channel mobility $\mu_{\text {eff }}$ model [6] by including carrier transport over $V_{g}$ modulated GB barrier

$$
\mu_{\text {eff }}=\mu_{\text {ig }} \exp \left(-S / V_{\text {gt }}\right) .
$$

$S$ is a constant relating to GB trap areal density [13]. It reflects GB barrier modulation effect and can be experimentally extracted.

Following gradual channel approximation (GCA), $I_{D}$ at a fixed $V_{D}$ is given by:

$$
I_{D}=W Q_{\mathrm{inv}}(y) v_{c}(y)
$$

where $v_{c}$ is carrier velocity at some point $y$ along the channel. Taking the velocity saturation effect into consideration, $v_{c}$ is modeled as [9], [14]

$$
v_{c}=\frac{\mu_{\mathrm{eff}} d V_{c} / d y}{1+\left(\mu_{\mathrm{eff}} / v_{\mathrm{sat}}\right) d V_{c} / d y}
$$

where $v_{\text {sat }}$ is the carrier saturation velocity. Combining all the above equations into (4), and integrating it from the source $y=0$ to the drain $y=L$, one obtains

$$
\begin{aligned}
I_{D} L=W C_{\mathrm{ox}} V_{\mathrm{gt}}^{2} \int_{0}^{V_{D} / V_{\mathrm{gt}}} \mu_{\mathrm{eff}}(1-x) d x-\frac{I_{D}}{v_{\mathrm{sat}}} \int_{0}^{V_{D}} \mu_{\mathrm{eff}} d V_{c} \\
x \equiv V_{c} / V_{\mathrm{gt}} .
\end{aligned}
$$

Define $\bar{\mu}_{\text {eff }} \equiv \int_{0}^{V_{D}} \mu_{\text {eff }} d V_{c} / V_{D}$, and the saturation field $E_{\text {sat }} \equiv$ $v_{\text {sat }} / \bar{\mu}_{\text {eff }}$, one has

$$
\begin{aligned}
I_{D}= & \frac{W}{L+V_{D} / E_{\mathrm{sat}}} C_{\mathrm{ox}} V_{\mathrm{gt}}^{2} \int_{0}^{V_{D} / V_{\mathrm{gt}}} \mu_{\mathrm{eff}}(1-x) d x \\
= & \frac{W}{L+V_{D} / E_{\mathrm{sat}}} C_{\mathrm{ox}} V_{\mathrm{gt}}^{2} \\
& \times \int_{0}^{V_{D} / V_{\mathrm{gt}}} \frac{\exp \left[-\left(S / V_{\mathrm{gt}}\right) /(1-x)\right]}{1+[\beta(1-x)]^{\nu}} \mu_{0}(1-x) d x
\end{aligned}
$$

where $\beta=\eta C_{\mathrm{ox}} V_{\mathrm{gt}} / \varepsilon_{s} E_{\text {crit }}$. Define $f(x)=\left(\exp \left[-\left(S / V_{\mathrm{gt}}\right) /\right.\right.$ $\left.(1-x)] / 1+[\beta(1-x)]^{\nu}\right)$, according to the mean value theorem for integration, there always exists an $x_{0} \in\left(0, V_{D} / V_{\mathrm{gt}}\right)$ so that $f\left(x_{0}\right)$ can be taken out from the integral. Therefore, $I_{D}$ is finally derived as

$$
\begin{aligned}
I_{D} & =\frac{W}{L+V_{D} / E_{\mathrm{sat}}} \bar{\mu}_{\mathrm{eff}} C_{\mathrm{ox}}\left(V_{\mathrm{gt}}-\frac{1}{2} V_{D}\right) V_{D} \\
\bar{\mu}_{\mathrm{eff}} & =\mu_{0} f\left(x_{0}\right), \quad x_{0}=\delta V_{D} / V_{\mathrm{gt}} \quad 0<\delta<1 .
\end{aligned}
$$

Parameter $\delta$ defines a position in the inversion channel where local mobility equals $\bar{\mu}_{\text {eff }}$. Thus, it could be named "average mobility parameter." This $I_{D}$ expression is similar to most forms for MOSFETs and TFTs, except for the modified mobility term. Three important mechanisms, namely, velocity

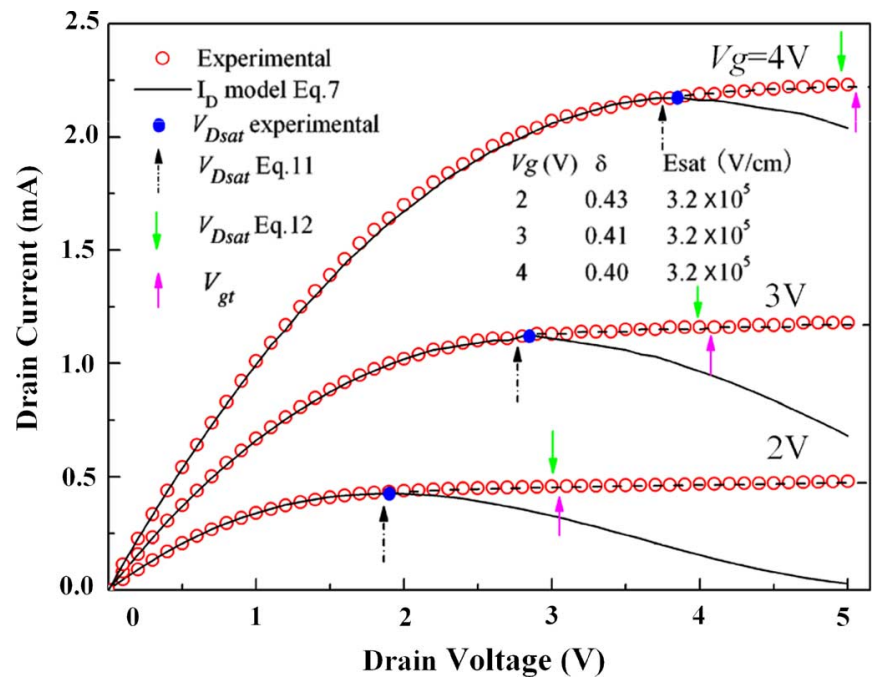

Fig. 1. $I_{D}$ model fit of output characteristics at various $V_{g}$ before saturation (solid lines) for an n-type high temperature processed poly-Si TFT. $V_{\text {Dsat }}$ calculated by (11) and (12), and $V_{\mathrm{gt}}$ are compared to the experimentally extracted $V_{\text {Dsat }}$. Dashed lines are fitting curves based on channel length modulation after saturation.

saturation, mobility degradation and GB barrier modulation are accurately incorporated. The derivation is purely based on GCA without any other approximations made.

\section{Drain Saturation Voltage}

The output characteristic is modeled based on the above $I_{D}$ model. At a fixed $V_{g}$, with increasing $V_{D}$ to $V_{\text {Dsat }}$, pinchoff occurs at the drain end. Drain-current wherein defines the saturation current $I_{\text {Dsat }}$, which still observes (7)

$$
I_{\text {Dsat }}=\frac{W}{L+V_{\text {Dsat }} / E_{\mathrm{sat}}} \bar{\mu}_{\mathrm{eff}} C_{\mathrm{ox}}\left(V_{\mathrm{gt}}-\frac{1}{2} V_{\text {Dsat }}\right) V_{\text {Dsat }} .
$$

By fitting the measured output curve at a given $V_{g}$ before saturation, $V_{\text {Dsat }}$ can be experimentally extracted as the $V_{D}$ position at which the fitted $I_{D}$ curve reaches maximum [9], as shown in Fig. 1. Apparently, this approach is not straightforward and $V_{\text {Dsat }}$ cannot be obtained without measuring and fitting each output curve. The accuracy of the method depends on the curve fitting.

To derive an analytical form for $V_{\text {Dsat }}$, let $\partial I_{D} / \partial V_{D}=0$, a quadratic equation of $V_{D}$ is obtained

$$
\begin{aligned}
& \frac{1}{2} \xi V_{D}^{2}-(\kappa+\xi) V_{\mathrm{gt}} V_{D}+V_{\mathrm{gt}}^{2}=0 \\
& \kappa=1+\frac{V_{\mathrm{gt}} / V_{D}-1 / 2}{L E_{\mathrm{sat}} / V_{D}+1}, \\
& \xi=\left(\delta+\frac{\partial \delta}{\partial V_{D}} V_{D}\right)\left[\frac{S}{V_{\mathrm{gt}}\left(1-\delta V_{D} / V_{\mathrm{gt}}\right)^{2}}\right. \\
& \left.+\frac{\nu \beta^{\nu}\left(1-\delta V_{D} / V_{\mathrm{gt}}\right)^{\nu-1}}{1+\beta^{\nu}\left(1-\delta V_{D} / V_{\mathrm{gt}}\right)^{\nu}}\right]
\end{aligned}
$$

$V_{\text {Dsat }}$ is the solution of (9)

$$
V_{\text {Dsat }}=\left(1+\kappa / \xi-\sqrt{(1+\kappa / \xi)^{2}-2 / \xi}\right) V_{\text {gt }} .
$$


TABLE I

$V_{\text {Dsat }}$ (IN volts) CALCUlated BASED on (10)-(12) ARE CoMPared TO EXPERIMENTALly EXTRACTED $V_{\text {Dsat }}$ AT VARIOUS $V_{g}$, FOR BOTH n- AND p-TYPE POLY-Si TFTs B Y EITHER LOW OR HIGH TEMPERATURE PROCESS

\begin{tabular}{|c|c|c|c|c|c|c|c|c|c|}
\hline \multirow{2}{*}{\multicolumn{2}{|c|}{$\frac{\text { Poly-Si TFTs }}{\text { Device threshold }}$}} & \multicolumn{2}{|c|}{ n-type HT } & \multicolumn{2}{|c|}{ n-type LT } & \multicolumn{2}{|c|}{ p-type HT } & \multicolumn{2}{|c|}{ p-type LT } \\
\hline & & & $64 \mathrm{~V}$ & & & & & & \\
\hline \multicolumn{2}{|c|}{$V_{g}(\mathrm{~V})$} & 2 & 4 & 8 & 10 & -3 & -5 & -15 & -18 \\
\hline \multicolumn{2}{|c|}{$\left|V_{\text {Dsat }}\right|$ extracted } & 1.89 & & 4.66 & & 1.29 & 02 & .79 & 22 \\
\hline \multicolumn{2}{|c|}{$\left|V_{\text {Dsat }}\right|$ Eq. 10} & 1.92 & & & & 1.30 & & 3.76 & \\
\hline \multicolumn{2}{|c|}{$\left|V_{\text {Dsat }}\right|$ Eq. $10 S=0$} & 2.97 & 4.77 & 8.05 & & 1.78 & & 9.08 & 11.1 \\
\hline \multirow{3}{*}{$\begin{array}{c}\left|V_{\text {Dsat }}\right| \\
\text { deviation } \\
(\%)\end{array}$} & $\delta=0$ & -3.70 & -7.25 & -2.79 & -3.77 & -2.33 & -4.30 & -6.90 & -8.36 \\
\hline & $\beta=0$ & 2.12 & 4.25 & 1.50 & 2.07 & 0.78 & 430 & 0.85 & 163 \\
\hline & & 0.53 & & 1.50 & & 1.55 & & 3.68 & 4.85 \\
\hline \multirow{2}{*}{$\begin{array}{l}\left|V_{\text {Dsat }}\right| \\
\text { Eq.11 }\end{array}$} & & 1.86 & & 4.66 & & 1.28 & & 3.69 & 0.09 \\
\hline & $\delta$ fixed & 1.91 & 3.6 & 4.66 & 6.2 & 1.23 & 3.11 & 4.15 & 6.16 \\
\hline \multicolumn{2}{|c|}{$\left|V_{\text {Dsat }}\right|$ Eq.12 } & 3.03 & 4.98 & 8.18 & 10.1 & 1.79 & 3.66 & 9.63 & 12.4 \\
\hline \multicolumn{2}{|c|}{$\left|V_{g t}\right|$} & 3.04 & 5.04 & 8.33 & 10.3 & 1.81 & 3.81 & 9.95 & 13.0 \\
\hline
\end{tabular}

In the above equation, dimensionless coefficient $\kappa$ is related to velocity saturation $\left(E_{\mathrm{sat}}\right) ; \xi$ is a combination of average mobility parameter $\delta$ with GB barrier modulation $(S)$ and mobility degradation effect $(\beta)$. Parameter $S$ is extracted as the slope of $\ln \mu_{\text {eff }}$ or $\ln \left(I_{D} / V_{\text {gt }}\right)$ versus $-1 / V_{\text {gt }}$ plot; and $E_{\text {crit }}$ is obtained by fitting the $V_{g}$ dependence of $\mu_{\text {eff }}$ [6]. $\delta$ and $E_{\text {sat }}$ are extracted by fitting the output curve before saturation. In addition, $\delta$ is weakly $V_{D}$ dependent and its differential $\delta^{\prime}$ is estimated by fitting transfer curves at various $V_{D}$. Since both coefficients are $V_{D}$ dependent, the $V_{\text {Dsat }}$ solution (10) is implicit and must be calculated through iteration. The value $V_{\mathrm{gt}}$ is ideal to be taken as the initial solution for the iteration. As listed in Table I, calculated $V_{\text {Dsat }}$ is within 1\% error compared to experimentally extracted value.

Since complicated procedures need to be followed to extract all parameters before $V_{\text {Dsat }}$ calculation, it is worth to observe which factor is dominant. In (10), neglect GB barrier modulation $(S=0), V_{D}$ dependence of $\delta\left(\delta^{\prime}=0\right)$, mobility degradation $(\beta=0)$ or velocity saturation $(\kappa=1)$, respectively, the corresponding $V_{\text {Dsat }}$ value/deviations are compared in the Table. Significant deviation caused by setting $S=0$ is in contrast to the small error introduced by the other three approximations. It clearly indicates that GB barrier modulation is the dominant factor deciding the $V_{\text {Dsat }}$. Interestingly, the overall error contributed by the three approximations can largely offset. Therefore, setting $\beta=0, \kappa=1$ and $\delta^{\prime}=0$ simultaneously, a much simplified $V_{\text {Dsat }}$ expression with reasonable accuracy is obtained

$$
\begin{aligned}
V_{\text {Dsat }} & =V_{\mathrm{gt}}\left(1+1 / \xi-\sqrt{1+1 / \xi^{2}}\right) \\
\xi & =\delta S V_{\mathrm{gt}} /\left(V_{\mathrm{gt}}-\delta V_{D}\right)^{2} .
\end{aligned}
$$

Although only GB effect is included and two parameters $S$ and $\delta$ involved in (11), the calculated $V_{\text {Dsat }}$ is within 3\% deviation from experimental values. $S$ is a GB related parameter independent of $V_{g}$, while $\delta$ still weakly depends on $V_{g}$. In modeling, $\delta$ can be accurately obtained by fitting the output curve at specific $V_{g}$. Fortunately, as an average mobility parameter, $\delta$ is nearly a constant. All $\delta$ values from 4 different types of TFTs are found to fall within $0.35 \sim 0.45$. For a certain kind of device, an average $\delta$ value from such device can be used for $V_{\text {Dsat }}$ prediction. As shown in the table, straightforward $V_{\text {Dsat }}$ prediction based on fixed $\delta$ value can typically have $4 \%$, or in the worst condition, $9 \%$ deviation. It should be acceptable for many practical applications. In (10), if only velocity saturation is included, i.e., $\xi=0$, the $V_{\text {Dsat }}$ solution reduces to a standard form for MOSFETs [9]

$$
\left.V_{\text {Dsat }}\right|_{\xi=0}=L E_{\text {sat }}\left(\sqrt{1+2 V_{\text {gt }} / L E_{\text {sat }}}-1\right)
$$

which could be further reduced to $V_{\mathrm{gt}}$, i.e., the $V_{\text {Dsat }}$ of ideal MOSFETs if $E_{\mathrm{sat}}=\infty$. As a comparison, calculated $V_{\text {Dsat }}$ using (12) is apparently erroneous for poly-Si TFTs, which actually is close to the value obtained by setting $S=0$ in (10).

\section{CONCLUSION}

For the first time, a physical-based full analytical expression for $V_{\text {Dsat }}$ of poly-Si TFTs is derived, which, after being simplified, can be used for straightforward $V_{\text {Dsat }}$ prediction with reasonable accuracy without measuring device output curves. The applicability of the formula is demonstrated by four different types of poly-Si TFTs.

\section{REFERENCES}

[1] J. D. Mark, S. S. Michael, and H. Michael, "Threshold voltage, field effect mobility, and gate-to-channel capacitance in polysilicon TFTs," IEEE Trans. Electron Devices, vol. 43, no. 9, pp. 1433-1440, Sep. 1996.

[2] M. Estrada, A. Cerdeira, A. Ortiz-Conde, F. J. Garcia Sanchez, and B. Iniguez, "Extraction method for polycrystalline TFT above and below threshold model parameters," Solid State Electron., vol. 46, no. 12, pp. 2295-2300, Dec. 2002.

[3] S. Chopra and R. S. Gupta, "Modeling of short geometry polycrystallinesilicon thin-film transistor," IEEE Trans. Electron Devices, vol. 47, no. 12, pp. 2444-2446, Dec. 2000.

[4] G.-Y. Yang, S.-H. Hur, and C.-H. Han, "A physical-based analytical turnon model of polysilicon thin-film transistors for circuit simulation," IEEE Trans. Electron Devices, vol. 46, no. 1, pp. 165-172, Jan. 1999.

[5] P.-S. Lin, J.-Y. Guo, and C.-Y. Wu, "A quasi-two-dimensional analytical model for the turn-on characteristics of polysilicon thin-film transistors," IEEE Trans. Electron Devices, vol. 37, no. 3, pp. 666-674, Mar. 1990.

[6] M. Wang and M. Wong, "An effective channel mobility based analytical on-current model for polycrystalline silicon thin-film transistors," IEEE Trans. Electron Devices, vol. 54, no. 4, pp. 869-874, Apr. 2007.

[7] A. L. Silburst, R. C. Foss, and W. F. Petrie, "An efficient MOS transistor model for computer-aided design," IEEE Trans. Comput.-Aided Design Integr. Circuits Syst., vol. CAD-3, no. 1, pp. 104-111, Jan. 1984.

[8] B. J. Sheu, D. L. Scharfetter, P.-K. Ko, and M.-C. Jeng, "BSIM: Berkeley short-channel IGFET model for MOS transistors," IEEE J. Solid-State Circuits, vol. SC-22, no. 4, pp. 558-567, Aug. 1987.

[9] N. Arora, MOSFET Models for VLSI Circuit Simulation Theory and Practice. New York: Springer-Verlag, 1993, ch. 6.

[10] M.-S. Liang, J. Y. Choi, P.-K. Ko, and C. Hu, "Inversion-layer capacitance and mobility of very thin gate-oxide MOSFETs," IEEE Trans. Electron Devices, vol. ED-33, no. 3, pp. 409-413, Mar. 1986.

[11] A. G. Sabnis and J. T. Clemens, "Characterization of the electron mobility in the inverted $\langle 100\rangle$ Si surface," in IEDM Tech. Dig., 1979, pp. 18-21.

[12] V. M. Agostinelli, Jr. and H. Shin, "A comprehensive model for inversion layer hole mobility for simulation of submicrometer MOSFETs," IEEE Trans. Electron Devices, vol. 38, no. 1, pp. 151-159, Jan. 1991.

[13] H.-L. Chen and C.-Y. Wu, "An analytical grain-barrier height model and its characterization for intrinsic poly-Si thin-film transistor," IEEE Trans. Electron Devices, vol. 45, no. 10, pp. 2245-2247, Jan. 1998.

[14] K. K. Thornber, "Relation of drift velocity to low-field mobility and highfield saturation velocity," J. Appl. Phys., vol. 51, no. 4, pp. 2127-2136, Apr. 1980. 